\title{
Effects of a multidisciplinary program on autonomic modulation in overweight or obese children and adolescents
}

\author{
Ana Laura Ricci-Vitor (MPT) ${ }^{1}$, Fabrício Eduardo Rossi (MPT)², Patricia Miyuki Hirai (BPT) ${ }^{1}$,

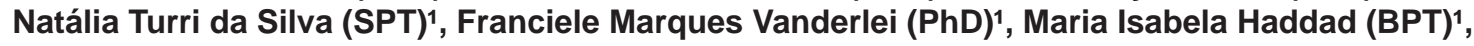 \\ Luana Almeida Gonzaga (BPT) ${ }^{1}$, Carlos Marcelo Pastre $(\mathrm{PhD})^{1}$, Vitor Engrácia Valenti (PhD) ${ }^{3}$, \\ Ismael Fortes Freitas Jr (PhD) ${ }^{2}$, Celso Ferreira (PhD) ${ }^{4}$, Luiz Carlos Marques Vanderlei (PhD) ${ }^{1}$
}

DOI: http://dx.doi.org/10.7322/jhgd.119257

\begin{abstract}
Purpose: this study evaluated the effects of a multidisciplinary program on autonomic modulation in overweight or obese children and adolescents.

Methods: fifteen individuals with $10,93 \pm 2,28$ years were submitted to autonomic evaluation using heart rate variability before and after a program based on physical exercises for three months, three times a week, 60 minutes per session and psychological and nutritional counseling. Data was analyzed using the paired t-test or Wilcoxon test and the level of significance was set at $\mathrm{P}<0.05$.

Results: multidisciplinary program was able to promote benefits in autonomic modulation observed thought temporal (SDNN: 39,96 \pm 10,33 x 49,44 $\pm 12,31 ; p=0,019 ;$ rMSSD: $28,97 \pm 11,50 \times 37,26$ $\pm 11,17 ; p=0,018$ ), spectral (LFnu: $64,49 \pm 12,21 \times 56,74 \pm 11,18 ; p=0,014 ;$ HFnu: $35,50 \pm 12,21 \times$ $43,25 \pm 11,18 ; p=0,014$ ) and geometrical (SD1: $20,51 \pm 8,13 \times 26,36 \pm 7,90 ; p=0,018$ SD2: $52,31 \pm$ $13,04 \times 64,58 \pm 16,33 ; p=0,031)$ indices in overweigh and obese children and adolescents. Also, after the intervention there was a reduction on resting heart rate $(88,53 \pm 9,24 \times 83,09 \pm 7,93 ; p=0,023)$ and there were changes in the classification of overweigh $26,67 \% \times 46,66 \%$ and obesity $73,33 \% \times 53,33 \%$, although not significant changes in body mass index.
\end{abstract}

Conclusions: exercise program together with nutritional and psychological counseling is able to promote benefits in autonomic modulation in children and adolescents who are overweight or obese.

Key words: autonomic nervous system, physical exercise, nutritional state, psychological counseling.

\section{INTRODUCTION}

Obesity is characterized as a major public health problem in children, as will be seen in the next generation $^{1}$. It has a multifactorial etiology influenced by genetic, behavioral and environmental factors ${ }^{2,3}$ and tends to persist into adult life $\mathrm{e}^{4}$. It is also associated with a number of complications such as some types of cancer $^{5}$, hypertension and dyslipidemias ${ }^{6}$.

Autonomic changes in obese children and adolescents are described in the literature ${ }^{7-9}$, as representing an important negative condition, since such changes are related to an increased risk of morbidity and mortality from several conditions ${ }^{10}$.

In this context, treatment of obese children and adolescents is of fundamental importance and physical activity, associated with a hypocaloric diet, is a tool that can promote a positive impact on this condition ${ }^{11}$. In this population, the practice of physical activity and nutritional intervention present evidence of benefits such as reduced adiposity, maintenance of stable weight and improvement of metabolic disorders and lipid use during maximum exercise ${ }^{12,13}$.

1 Departamento de Fisioterapia, Universidade Estadual Paulista (UNESP), Julio de Mesquita Filho, Presidente Prudente, São Paulo, Brasil.

2 Departamento de Educação Física, Universidade Estadual Paulista (UNESP), Julio de Mesquita Filho, Presidente Prudente, São Paulo, Brasil.

3 Departamento de Fonoaudiologia, Universidade Estadual Paulista (UNESP), Julio de Mesquita Filho, Marília, São Paulo, Brasil.

4 Departamento de Medicina (Cardiologia), Universidade Federal São Paulo (UNIFESP), São Paulo, São Paulo, Brasil.

Corresponding Author: Ana Laura Ricci-Vitor - E-mail: analaura.ricci@yahoo.com/lcmvanderlei@fct.unesp.br

Suggested citation: Ricci-Victor AL, Rossi FE, Hirai PM, Silva NT, Vanderlei FM, Haddad MI (et al). Effects of a multidisciplinary program on autonomic modulation in overweight or obese children and adolescents. J Hum Growth Dev. 26(1): 154-161. Doi: http://dx.doi. org/10.7322/jhgd.119257

Manuscript submitted: Jun 26 2016, accepted for publication Jul 212016. 
Some studies focusing on children have stressed the beneficial effects of physical activities on autonomic modulation. Gutin et al. ${ }^{14}$, after a program of physical exercise with 7- to 11-year-old obese children, saw an improvement in autonomic modulation, characterizing the program as capable of promoting an adequate stimulus for the cardiovascular system. Moreover, Nagai et al. ${ }^{15}$ observed that physical activity levels are related to autonomic changes in obese children and suggested that physical activity programs could contribute to the improvement of autonomic modulation in these children.

However, no studies were found that evaluate the effects of multidisciplinary treatments involving physical activity and nutritional and psychological counseling on autonomic modulation of children and adolescents above normal weight. The hypothesis is that this type of intervention could promote beneficial effects on the autonomic modulation of this population.

In order to insert new elements on this theme into the literature, this study aimed to evaluate the influence of a multidisciplinary program, combining exercise and nutritional and psychological counseling, on autonomic modulation in overweight or obese children and adolescents.

\section{METHODS}

\section{Subjects}

This study is a longitudinal clinical trial that evaluated a program based on physical exercises and nutritional and psychological counseling. It involved 38 overweight and obese children and adolescents of both genders, between 6 and 17 years of age, who reported no use of medicine or cardiorespiratory and metabolic diseases. Among them, 23 (60.52\%) were excluded because they abandoned the three month exercise protocol, the final sample thus consisted of 15 volunteers, seven males (46.66\%) and eight females (53.33\%). The characteristics of the final sample are presented in table 1

\section{Ethical procedures}

The volunteers and their parents/guardians received an explanation of the objectives and procedures of the study and signed an informed consent form. The procedures used in this study were approved by the Research Ethics Committee of the Faculty of Sciences and Technology, Paulista State University, Presidente Prudente, São Paulo, Brazil, (Protocol n ${ }^{\circ}$ 259/2008) and followed resolution 196/96 of the National Health Council.

\section{Study design}

The experimental protocol consisted of an initial evaluation, a multidisciplinary program and a final evaluation. During the initial evaluation, anamnesic, anthropometric and autonomic evaluations were conducted. Following this, the participants took part in the multidisciplinary program and, on completion, the final evaluation, including anthropometric and autonomic evaluations, was conducted. The structure of these methods was based on the Consolidated Standards of Reporting Trials (CONSORT) guidelines which can be found at: www.consort-statement.org/.

\section{Medical history and anthropometry}

Personal information on the volunteers, together with their complete medical history, were recorded, with questions regarding the use of medicine and cardiorespiratory and metabolic diseases in order to determine whether they fulfilled the inclusion criteria. Anthropometry measurements were performed to determine their compliance with the targeted population. Body weight and height were measured and the body mass index (BMI) was calculated using the following formula: weight (kilograms$\mathrm{Kg}$ )/height ${ }^{2}$ (meters-m). Weight was measured on a digital scale (Welmy, R/I 200, Brazil), with a maximum capacity of $180 \mathrm{~kg}$ and a precision of $0.1 \mathrm{~kg}$, with the volunteers in an orthostatic position, barefoot and wearing light clothing. Height was measured using a stadiometer (Sanny, Brazil), with a precision of $0.1 \mathrm{~cm}$ and a maximum length of $2.20 \mathrm{~m}^{16}$. From the BMI, the volunteers were classified as overweight or obese according to sex and age following the criteria proposed by Cole et al. ${ }^{17}$.

\section{Autonomic modulation}

Autonomic modulation was assessed in the morning between 8.00 and 12.00 to minimize the influence of circadian rhythm, in a temperature-controlled room of between $21^{\circ} \mathrm{C}$ and $23{ }^{\circ} \mathrm{C}$ and humidity between $40 \%$ and $60 \%$. The volunteers were instructed to avoid stimulant drinks such as coffee, tea and soft drinks and/or food containing chocolate for a period of 12 hours prior to the autonomic evaluation.

During the autonomic evaluation, the volunteers were instructed to remain awake, in silence, breathing spontaneously while resting in the supine position for 20 minutes on a couch. After receiving an explanation of the data collection procedures, an electrode was placed on the volunteer's chest next to the xiphoid process using an elastic strap, and a heart rate receiver (Polar Electro, model S810i, Finland) was attached to the participant's wrist. The equipment had been previously validated to record beat-to-beat heart rate and for use in collecting heart rate variability (HRV) data for analysis ${ }^{18}$.

The behavioral pattern was registered beat-to-beat with a sampling rate of $1000 \mathrm{~Hz}$ for HRV analysis. Thousand intervals of consecutive cardiac beats were used to analyze HRV indices, selected after digital filtering, complemented by manual filtering to eliminate artifact and ectopic beats, and only series with more than 95\% sinus beats were included in the study.

The HRV analysis was performed through the linear indices of the time (rMSSD and SDNN) and frequency domains (LF, HF, LF/HF) and the geometric indices: triangular index (RRtri), TINN and Poincaré plot (SD1, SD2, SD1/SD2). In the time domain, the following indices were used: rMSSD (the root mean square of successive differences between normal intervals of consecutive cardiac beats in an interval of time expressed in milliseconds [ms]) and SDNN (the standard deviation of normal-to-normal intervals in an interval of time expressed in $\mathrm{ms})^{19}$.

In the frequency domain, the spectral components of low frequency (LF:0.004 to 0.15 Hertz [Hz]) and high frequency (HF: 0.15 to $0.40 \mathrm{~Hz}$ ) were used, expressed in 
$\mathrm{ms}^{2}$ (ms squared) and normalized units (nu), which represent the relative value of each spectral component at the total potency minus the very low frequency, and the ratio between them $(\mathrm{LF} / \mathrm{HF})^{19}$. These analyses were calculated using the Fast Fourier Transform algorithm.

The following geometric indices used were: RRtri, TINN and Poincaré plot. RRtri was calculated from the density histogram of iRR and consisted of the integral of the histogram (i.e., the total number of iRR) divided by the maximum of the density distribution (modal frequency of iRR), measured on a discrete scale with $7.8125 \mathrm{~ms}$ boxes (1/128 seconds). The TINN index consisted of the base width of the distribution measured as the base of a triangle, approximating the distribution of all iRR, the minimum square difference being used to determine the triangle. The Poincaré plot allowed each iRR to be plotted against the previous interval. The duration of an interval (RRn) was represented on the " $x$ " axis, and the length of the next interval $(R R n+1)$ on the " $y$ " axis, so each point on the graph (RRn, $R R n+1)$ corresponded to two successive heart beats. For quantitative analysis of the plot, the indices calculated were: SD1 (standard deviation of instantaneous beat to beat variability), SD2 (standard deviation of long term continuous iRR) and the SD1/SD2 ratio ${ }^{19}$.

The qualitative analysis of the plot was performed by analyzing the figures formed by its attractor, based on the description in Tulppo et al. ${ }^{20}$, as: I) a figure with a greater dispersion of the iRR beat-to-beat featuring a plot with greater variability in the long term and II) a figure with small overall dispersion beat-to-beat without increasing the dispersion of iRR in the long term, featuring a plot with less variability.

The analysis was processed using Kubios software (Biosignal Analysis and Medical Image Group, Department of Physics, University of Kuopio, Finland) ${ }^{21}$.

\section{Intervention program}

The developed program, entitled Super-Action, aims to treat children and adolescents through physical activities associated with psychological and nutritional counseling. The activities were conducted over a period of three months with a frequency of three times a week, 60 minutes per session. The volunteers were divided into two groups: children from 6 to 11 years and adolescents from
12 to 17 years, in order to ensure they carried out activities appropriate to their age group.

In relation to physical activity, each session consisted of five minutes of stretching at the beginning and end, 50 minutes of predominantly aerobic activities and exercises to stimulate the development of strength and resistance of large muscle groups (arms, legs, chest, shoulder, back and hip) in the form of walking and circuit activities. The activities had a playful and dynamic profile.

The nutritional and psychological interventions were carried out biweekly through group meetings at which instructions about healthy eating habits and psychological aspects of care were transmitted simply and clearly to the children together with their parents. This was done through tips on daily living and counseling in order to stimulate beneficial changes in lifestyle.

\section{Statistical analysis}

Characteristic data of the population were described as mean, standard deviation, median, minimum, maximum or absolute values and percentages. Data distribution was checked using the Shapiro-Wilk test to compare HRV indices before and after the intervention program, and, depending on the data distribution, the paired t-test or Wilcoxon test was used. Statistical analysis was performed using the SPSS statistical software package, version 13.00 (SPSS, Inc. Chicago, IL, USA). The level of statistical significance was set at $\mathrm{P}<0.05$ for all analyses.

\section{RESULTS}

The characteristics of the sample before and after the intervention are presented in Table 01. There were changes, although not significant in BMI, but in the classification of overweight and obesity. At the beginning of the protocol, 4 volunteers (26.67\%) were considered overweight and 11 (73.33\%) obese and at the end of the protocol three volunteers from the obese group had moved to the overweight group, totaling, seven volunteers overweight (46.66\%) and eight obese (53.33\%).

The values of the temporal indices of HRV are presented in Table 02

Table 1: Sample feature before and after the intervention at mean, standard deviation, median, minimum and maximum.

\begin{tabular}{cccc} 
Feature & Pre $(\mathbf{n}=\mathbf{1 5})$ & Post $(\mathbf{n}=\mathbf{1 5})$ & $\mathbf{p}$ \\
Age (years) & $10,93 \pm 2,28(11,00)$ & $11,33 \pm 2,19(11,00)$ & 0,0086 \\
& {$[7,00-15,00]$} & {$[8,00-15,00]$} & 0,2676 \\
Body mass (kg) & $69,80 \pm 34,26(58,20)$ & $70,66 \pm 32,79(58,90)$ & 0,0234 \\
Height $(\mathbf{m})$ & {$[39,70-154,90]$} & {$[42,10-148,90]$} & 0,4212 \\
& $1,51 \pm 0,14(1,46)$ & $1,55 \pm 0,14(1,58)$ & {$[1,32-1,76]$} \\
\hline
\end{tabular}

Legend: $\mathrm{p}=$ significance level, $\mathrm{BMI}=$ body mass index, $\mathrm{kg}=$ kilograms, $\mathrm{m}^{2}=$ squared meters . 
Table 2: Temporal indexes of heart rate variability express at mean, standard deviation, median and confidence interval.

\begin{tabular}{cccc} 
Indexes & Pre $(\mathbf{n}=\mathbf{1 5})$ & Post $(\mathbf{n}=\mathbf{1 5})$ & $\mathbf{p}$ \\
MeanRR(ms) & $685,98 \pm 74,10(677,30)$ & $730,74 \pm 74,55(41,50)$ & 0,024 \\
& {$[644,94-727,02]$} & {$[689,45-772,03]$} & 0,023 \\
MeanHR(bpm) & $88,53 \pm 9,24(88,98)$ & $83,09 \pm 7,93(83,01)$ & 0,019 \\
SDNN (ms) & {$[83,41-93,65]$} & {$[78,70-87,48]$} & 0,018 \\
\hline
\end{tabular}

Statistically significant difference by Student $t$ test for paired data $(p<0.05)$. Legend: $p=$ significance level, MeanRR $=$ mean of intervals between consecutive heart beats, MeanHR = mean of heart rate, SDNN = standard deviation of all normal to normal intervals, rMSSD = the square root of the mean of the sum of the squares of differences between adjacent normal to normal intervals, $\mathrm{ms}=$ milliseconds, $\mathrm{bpm}=$ beats per minute.

A significant increase in iRR mean, SDNN and rMSSD and a significant reduction in heart rate value were observed after the intervention.
The autonomic modulation data verified by the spectral indices are presented in Table 03. There was a significant increase in LH and HF obtained in $\mathrm{ms}^{2}$. When

Table 3: Spectral indexes of heart rate variability express at mean, standard deviation, median and confidence interval

\begin{tabular}{cccc}
\hline Indexes & Pre $(\mathbf{n}=\mathbf{1 5})$ & Post $(\mathbf{n}=\mathbf{1 5})$ & $\mathbf{p}$ \\
LF $\left(\mathbf{m s}^{2}\right)$ & $592,26 \pm 288,10(613,00)$ & $762,26 \pm 376,65(656,00)$ & 0,081 \\
& {$[432,71-751,83]$} & {$[553,67-970,87]$} & 0,018 \\
HF $\left(\mathbf{m s}^{2}\right)$ & $392,33 \pm 325,23(283,00)$ & $617,66 \pm 365,37(486,00)$ & 0,014 \\
& {$[212,21-572,46]$} & {$[415,31-820,02]$} & 0,014 \\
LF (nu) & $64,49 \pm 12,21(63,20)$ & $56,74 \pm 11,18(55,70)$ & $0,020^{\dagger}$ \\
\hline
\end{tabular}

Statistically significant difference by Student $t$ test for paired data $(p<0.05)$. †Statistically significant difference by Wilcoxon test $(p<0.05)$. Legend: $p=$ significance level, $L F=$ low frequency component, $\mathrm{HF}=$ high frequency component, $\mathrm{ms}^{2}=$ squared milliseconds, $\mathrm{nu}=$ normalized units.

measured in nu, there was a reduction in LF and an increase in HF. There was also a reduction in the LF/HF ratio.

The quantitative analysis of geometric indices of HRV is presented in Table 04 and the qualitative analysis in Figure 01. Regarding the quantitative analysis of geometric indices, there was a significant increase in TINN, SD1 and SD2. There were no changes to the RRtri and SD1/SD2 indices. There was greater dispersion of beatto-beat $\mathrm{iRR}$ in the qualitative analysis and in the long term after the multidisciplinary program compared with the pattern observed initially. Figure 01 demonstrates a pattern example of a Poincaré plot before (Figure 1A) and after (Figure 1B) the intervention.

\section{DISCUSSION}

The results of this study suggest that a program of physical exercise associated with nutritional and psycho- logical counseling can have a positive influence on autonomic modulation in children and adolescents who are overweight or obese. Additionally, there was a reduction in resting heart rate and changes in the classification category of some children after the intervention from obese to overweight, although there was no significant difference in BMI.

Regarding the temporal and spectral indices of HRV which represent the parasympathetic autonomic nervous system (rMSSD, HF (ms²), and SD1) ${ }^{19}$, there was an increase in values when comparing the moments before and after the intervention. There is evidence that obese children demonstrate reduced parasympathetic activity ${ }^{7,22}$, which is an important negative factor as this reduction is associated with the development of risk factors and an increase in morbidity and mortality ${ }^{23}$. Thus, the increase in parasympathetic activity induced by the applied intervention program represents an important aspect for these children. 
Table 4: Geometric indexes of heart rate variability express at mean, standard deviation, median and confidence interval

\begin{tabular}{|c|c|c|c|}
\hline Indexes & Pre $(n=15)$ & Post $(n=15)$ & p \\
\hline \multirow{3}{*}{ TINN(ms) } & $188,33 \pm 45,65(190,00)$ & $225,33 \pm 45,01(220,00)$ & \multirow{3}{*}{0,014} \\
\hline & & & \\
\hline & {$[163,05-213,62]$} & {$[200,40-250,27]$} & \\
\hline \multirow{3}{*}{ RRtri } & $10,87 \pm 2,91(11,11)$ & $11,97 \pm 3,02(12,05)$ & \multirow{3}{*}{0,170} \\
\hline & & & \\
\hline & {$[9,25-12,49]$} & {$[10,29-13,64]$} & \\
\hline \multirow{3}{*}{ SD1(ms) } & $20,51 \pm 8,13(20,70)$ & $26,36 \pm 7,90(26,60)$ & \multirow[b]{2}{*}{0,018} \\
\hline & {$[16,00-25,01]$} & {$[21,98-30,74]$} & \\
\hline & $52,31 \pm 13,04(55,10)$ & $64,58 \pm 16,33(62,10)$ & \multirow[b]{2}{*}{$0,031^{\dagger}$} \\
\hline $\mathrm{SD2}(\mathrm{ms})$ & {$[45,08-59,53]$} & {$[55,53-73,62]$} & \\
\hline SD1/SD2 & $0,38 \pm 0,10(0,37)$ & $0,40 \pm 0,08(0,42)$ & 0,364 \\
\hline
\end{tabular}

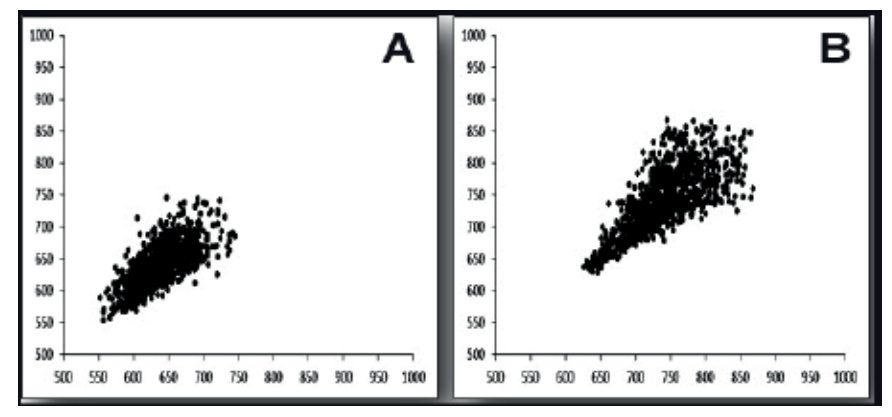

Figure 01. Visual pattern of Poincaré plot before (1A) and after (1B) intervention.

The temporal and spectral indices of HRV which represent overall variability (SDNN, SD2, TINN and RRtri), and the LF (ms²) index which shows the sympathetic component of the autonomic nervous system ${ }^{19}$, also increased after implementing the intervention program. The literature highlights evidence of reduced sympathetic activity in obese children ${ }^{7,22}$ which could represent another negative aspect in these children as the sympathetic nervous system participates in the modulation of glucose and lipid metabolism ${ }^{24}$ and, thus, a reduction in sympathetic activity is associated with less energy expenditure and consequent weight gain ${ }^{25}$.

Regarding the analysis of the spectral indices expressed in nu, there was an improved balance in the ratio between the sympathetic and parasympathetic components of the ANS, with an increase in parasympathetic activity relative to sympathetic activity. The normalization process could minimize the effects of changes in the very low frequency band of the power spectrum and is determined by the division of the low frequency or high frequency power components by the total power spectrum, minus the very low frequency component, multiplied by $100^{19}$.

Regarding the SD1/SD2 ratio, no significant differences were observed between the pre- and post-intervention moments, which could be explained by the increase in both SD1 and SD2 indices after the completion of the intervention program.

The qualitative analysis of the Poincaré plot suggests that the applied program promoted an increase in HRV and hence improved autonomic modulation. After the intervention, the figure of the Poincaré plot showed higher dispersion of beat-to-beat and long term, featuring a plot with more variability when compared to the preintervention.

Additionally, the program positively influenced the classification of children according to BMI, since some of the children went from being obese to being overweight. Kelley \& Kelley et al. ${ }^{26}$, showed that exercise is effective 
in reducing the percentage of body fat in obese children and adolescents, although not sufficiently to suggest that exercise reduces other measures of adiposity.

In this study, there was an average reduction of five beats per minute (6.14\%) in heart rate after the intervention. Reduction in heart rate in obese adolescents aged 13 to 16 years after three months of interval aerobic training has been reported in the literature ${ }^{27}$ and, independent of obesity, adolescent athletes have a bradycardia response resulting from physical exercise when compared to non-athletes ${ }^{28}$.

As reported above, obese children and adolescents demonstrated changes in autonomic behavior of the nervous system characterized by reductions in both sympathetic and parasympathetic activity ${ }^{7,22}$, which is associated with higher risks of morbidity and mortality ${ }^{23}$. Given this and the epidemiological importance of obesity ${ }^{1}$, any intervention capable of inducing improved autonomic modulation is fundamental and should be implemented.

The physiological mechanisms responsible for these changes have not yet been described and studies are needed in order to understand them. Regarding exercise, it is known that a program of physical activity performed three times a week, for three months in obese pre-pubertal children can decrease blood pressure, arterial stiffness and abdominal fat; increase cardiorespiratory functional capacity and delay arterial wall remodeling ${ }^{29}$; and can even promote improved autonomic modulation in both obese ${ }^{15}$ and healthy children ${ }^{14}$.

Evidence regarding the benefits of nutritional counseling $^{19}$, and psychological treatments ${ }^{30}$ pointed to positive influences on obesity in children, but information about the effects of such interventions on autonomic modulation are unknown.

The association of physical exercise and a hypocaloric diet together are known to produce a positive impact on obesity ${ }^{19}$, and in obese children, there is evidence of benefit in reduced adiposity, maintenance of stable weight and improvement in metabolic disorders and lipid use dur- ing maximum exercises ${ }^{12-13}$.

Among the limitations of this study, it is worth pointing out that $60.52 \%$ of the subjects did not complete the three-month program, thus reducing the sample size and as a result, the analyses of gender, age and influence of maturational stage were not carried out in relation to the HRV. Furthermore, the intervention time (12 weeks) may have been insufficient to promote reduction in the body weight of the participants.

It is therefore concluded that an exercise program together with nutritional and psychological counseling is able to promote benefits in autonomic modulation in children and adolescents who are overweight or obese, which reinforces the importance of multidisciplinary treatment of childhood obesity, as an efficient tool for reversing the physiological changes caused by obesity.

\section{Contribution in public health}

It is known that childhood obesity will be the major public health problem to be seen in the next generation. Also, an important fact is that this disease is likely to persist into adult life and be associated with a number of complications such as cardiovascular diseases, which remain a leading cause of death in Brazil and in the world, hypertension; dyslipidemias; diabetes and even cancer. In consequence, this leads to a social and economic burden. This study is about a multidisciplinary treatment, based on nutritional and psychological guidance along with a physical exercise program in children and youth who are overweight or obese. Interventions contributing to the treatment of obesity may, in the future, contribute to reducing the cost of treating the health complications as well as the social burden related to obesity.

\section{Author Disclosures}

The authors declare that they have no conflict of interest and would like to thank Christopher R. Shoebridge for the English review of this paper.

\section{REFERENCES}

1. Lobstein T, Baur L, Uauy R. Obesity in children and young people: a crisis in public health. Obes Rev. 2004;5(Suppl.1):4-85. DOI: http://dx.doi.org/10.1111/j.1467-789X.2004.00133.x

2. Karnik S, Kanekar A. Childhood obesity: a global public health crisis. Int J Prev Med. 2012; 3(1):1-7. DOI: http://dx.doi.org/10.1201/b18227-3

3. Moraeus L, Lissner L, Yngve A, Poortvliet E, Al-Ansari U, Sjöberg A. Multi-level infl uences on childhood obesity in Sweden: societal factors, parental determinants and child's lifestyle. Int J Obes (Lond). 2012;36(7): 969-76. DOI: http://dx.doi.org/10.1038/ijo.2012.79

4. Abrantes MM, Lamounier JA, Colosimo EA. Prevalência de sobrepeso e obesidade em crianças e adolescentes das regiões sudeste e nordeste. J Pediatr (Rio J). 2002;78(4):335-40. DOI: http://dx.doi. org/10.1590/S0021-75572002000400014

5. Arem H, Irwin ML. Obesity and endometrial cancer survival: a systematic review. Int J Obes (Lond). 2013;37(5):634-9. DOI: http://dx.doi.org/10.1038/ijo.2012.94

6. Centers for Disease Control and Prevention (CDC). Overweight and obesity; childhood overweight and obesity, consequences. [cited 2009 Oct 08]. Available from: http://www.cdc.gov/obesity/childhood/consequences.html. 
7. Souza NM, Rossi RC, Vanderlei FM, Ricci-Vitor AL, Bernardo AFB, Gonçalves ACCR, et al. Variabilidade da frequência cardíaca em crianças obesas. Journal of Human Growth and Development. 2012;23(2): 328-333. DOI: http://dx.doi.org/10.7322/jhgd.46456

8. Guízar JM, Ahuatzin R, Amador N, Sánchez G, Romer G. Heart Autonomic Function in Overweight Adolecents. Indian Pediatr. 2005;42(5):464-9.

9. Vanderlei FM, Vanderlei LCM, Gardner DM. Heart rate dynamics by novel chaotic globals to hrv in obese youths. J Hum Growth Dev. 2015;25(1):82-8. DOI: http://dx.doi.org/10.7322/jhgd.96772

10. Godoy MF, Takakura IT, Correa PR. Relevância da análise do comportamento dinâmico não-linear (Teoria do Caos) como elemento prognóstico de morbidade e mortalidade em pacientes submetidos à cirurgia de revascularização miocárdica. Arq Ciênc Saúde. 2005;12(4):167-71.

11. Waters E, Silva-Sanigorski A, Burford BJ, Brown T, Campbell KJ, Gao Y, et al. Interventions for preventing obesity in children. Sao Paulo Med J. 2014; 132(2):128-9. DOI: http://dx.doi.org/10.1590/15163180.20141322T2

12. Maffeis C, Castellani M. Physical activity: An effective way to control weight in children? Nut Metab Cardiovasc Dis. 2007;17(5):394-408. DOI: http://dx.doi.org/10.1016/j.numecd.2006.08.006

13. Ounis OB, Elloumi M, Amrib M, Trabelsi Y, Lac G, Tabka Z. Impact of training and hypocaloric diet on fat oxidation and body composition in obese adolescents. Sci Sports. 2009; 24(3-4):178-85. DOI: http:// dx.doi.org/10.1016/j.scispo.2008.10.002

14. Gutin B, Owens S, Slavens G, Riggs S, Treiber F. Effect of physical training on heart-period variability in obese children. J Pediatr. 1997;130(6):938-43.

15. Nagai N, Hamada T, Kimura T, Moritani T. Moderate physical exercise increases cardiac autonomic nervous system activity in children with low heart rate variability. Childs Nerv Syst. 2004;20(4):209-14. DOI: http://dx.doi.org/10.1007/s00381-004-0915-5

16. Lohman TG, Roche AF, Martorell R. Anthropometric standardization reference manual. Champaign: Human Kinetics Books; 1988.

17. Cole TJ, Bellizzi MC, Flegal KM, Dietz WH. Establishing a standard defi nition for child overweight and obesity worldwide: international survey. BMJ 2000;320:1240 DOI: http://dx.doi.org/10.1136/bmj.320.7244.1240

18. Vanderlei LCM, Silva RA, Pastre CM, Azevedo FM, Godoy MF. Comparison of the Polar S810i monitor and the ECG for the analysis of heart rate variability in the time and frequency domains. Braz $\mathrm{J}$ Med Biol Res. 2008;41(10):854-9. DOI: http://dx.doi.org/10.1590/S0100-879X2008005000039

19. Vanderlei LCM, Pastre CM, Hoshi RA, Carvalho TD, Godoy MF. Noções básicas de variabilidade da frequência cardíaca e sua aplicabilidade clínica. Rev Bras Cir Cardiovasc. 2009;24(2):205-17. DOI: http:// dx.doi.org/10.1590/S0102-76382009000200018

20. Tulppo MP, Makikallio TH, Takala TES, Seppanen T, Huikuri HV. Quantitative beat-to-beat analysis of heart rate dynamics during exercise. Am J Physiol. 1996;271(7):H244-52.

21. Tarvainen MP, Niskanen JP, Lipponen JA, Ranta-Ahoa PO, Karjalainen PA. Kubios HRV: Heart rate variability analysis software. Comput Methods Programs Biomed. 2014;113(1):210-20. DOI: http://dx.doi. org/10.1016/j.cmpb.2013.07.024

22. Nagai N, Matsumoto T, Kita H, Moritani T. Autonomic nervous system activity and the state and development of obesity in japanese school children. Obes Res. 2003;11(1):25-32. DOI: http://dx.doi.org/10.1038/ oby. 2003.6

23. Thayer JF, Lane RD. The role of vagal function in the risk for cardiovascular disease and mortality. Biol Psychol. 2007;74(2): 224-42. DOI: http://dx.doi.org/10.1016/j.biopsycho.2005.11.013

24. Nonogaki K. New insight into sympathetic regulation of glucose and fat metabolism. Diabetologia. 2000;43(5):533-49. DOI: http://dx.doi.org/10.1007/s001250051341

25. Peterson HR, Rothschild M, Weinberg CR, Fell RD, McLeish KR, Pfeifer MA. Body fat and the activity of the autonomic nervous system. N Engl J Med. 1988; 318(17):1077-83. DOI: http://dx.doi.org/10.1056/ NEJM198804283181701

26. Kelley GA, Kelley KS. Effects of Exercise in the Treatment of Overweight and Obese Children and Adolescents: A Systematic Review of Meta-Analyses. J Obes. 2013;2013. DOI: http://dx.doi. org/10.1155/2013/783103

27. Ingul CB, Tjonna AE, Stolen TO, Stoylen A, Wisloff U. Impaired cardiac function among obese adolescents: effect of aerobic interval training. Arch Pediat Adolesc Med. 2010;164(9):852-9. DOI: http://dx.doi. org/10.1001/archpediatrics.2010.158

28. Alom MM, Bhuiyan NI, Hossain MM, Hoque MF, Rozario RJ, Nessa W. Physical training induced resting bradycardia and its association with cardiac autonomic nervous activities. Mymensingh Med J. 2011;20(4):665-70.

29. Farpour-Lambert NJ, Aggoun Y, Marchand LM, Martin XE, Herrmann FR, Beghetti M. Physical activity reduces systemic blood pressure and improves early markers of atherosclerosis in pre-pubertal obese children. J Am Coll Cardiol. 2009;54(25):2396-2406. DOI: http://dx.doi.org/10.1016/j.jacc.2009.08.030

30. Daley AJ, Copeland RJ, Wright NP, Roalfe A, Wales JK. Exercise therapy as a treatment for psychopathologic conditions in obese and morbidly obese adolescents: a randomized, controlled trial. Pediatrics. 2006;118(5):2126-34. DOI: http://dx.doi.org/10.1542/peds.2006-1285. 
This article is distributed under the terms of the Creative Commons Attribution 4.0 International License (http://creativecommons.org/licenses/by/4.0/), which permits unrestricted use, distribution, and reproduction in any medium, provided you give appropriate credit to the original author(s) and the source, provide a link to the Creative Commons license, and indicate if changes were made. The Creative Commons Public Domain Dedication waiver (http://creativecommons.org/publicdomain/zero/1.0/) applies to the data made available in this article, unless otherwise stated.

\section{Resumo}

Objetivo: avaliar a influência de um programa multidisciplinar na modulação autonômica de crianças e adolescentes com sobrepeso e obesidade.

Método: quinze indivíduos com 10,93 $\pm 2,28$ anos foram submetidos a avaliação da modulação autonômica por meio da variabilidade da frequência cardíaca antes e após um programa de atividades físicas conduzido por três meses, com frequência de três vezes na semana por 60 minutos cada sessão, associado com aconselhamento nutricional e psicológico. Para análise estatística foi utilizado teste $T$ para dados pareados ou teste de Wilcoxon com significância de $p<0,05$.

Resultados: o programa multidisciplinar foi capaz de influenciar positivamente a modulação autonômica o que pode ser observado por índices temporais (SDNN = 39,96 $\pm 10,33$ vs. 49,44 $\pm 12,31$; $p=0,019$; rMSSD $=28,97 \pm 11,50$ vs. $37,26 \pm 11,17 ; p=0,018$ ), espectrais (LFnu $=64,49 \pm 12,21$ vs. $56,74 \pm 11,18 ; p=0,014 ; \mathrm{HFnu}=35,50 \pm 12,21$ vs. $43,25 \pm 11,18 ; p=0,014)$ e geométricos (SD1 = $20,51 \pm 8,13$ vs. $26,36 \pm 7,90 ; p=0,018 ;$ SD2 $=52,31 \pm 13,04$ vs. $64,58 \pm 16,33 ; p=0,031$ ) de variabilidade em crianças e adolescentes com sobrepeso e obesidade. Adicionalmente, após a intervenção houve redução da frequência cardíaca de repouso $(88,53 \pm 9,24$ vs. 83,09 $\pm 7,93 ; p=0,023)$ e mudanças na classificação da obesidade dos indivíduos para sobrepeso $26,67 \%$ vs. $46,66 \%$ e obesidade $73,33 \%$ vs. 53,33\%. Apesar disso, não houve diferença significante no índice de massa corporal.

Conclusões: um programa de exercícios associado com aconselhamento nutricional e psicológico foi capaz de promover benefícios na modulação autonômica em crianças e adolescentes com sobrepeso e obesidade.

Palavras-chave: sistema nervoso autônomo, exercício físico, estado nutricional e aconselhamento psicológico. 